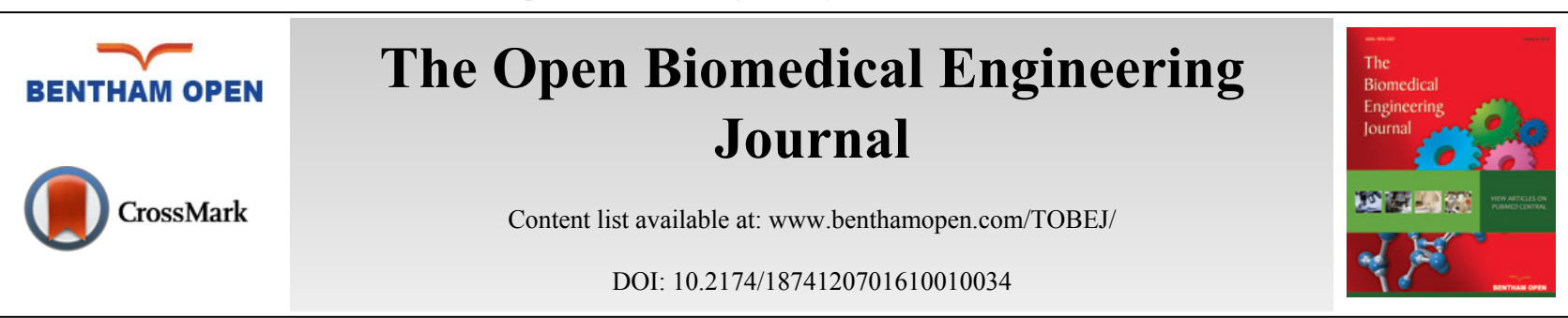

\title{
Ex Vivo Dermis Mechanical Behavior in Relation to Decellularization Treatment Length
}

Mara Terzini ${ }^{1}$, Cristina Bignardi ${ }^{1}$, Carlotta Castagnoli ${ }^{3}$, Irene Cambieri ${ }^{2}$, Elisabetta M. Zanetti ${ }^{3, *}$ and Alberto L. Audenino ${ }^{1}$

${ }^{1}$ Department of Mechanical and Aerospace Engineering, Politecnico di Torino, Torino, Italy

${ }^{2}$ Skin Bank, AOU Città della Salute e della Scienza, Torino, Italy

${ }^{3}$ Department of Engineering, University of Perugia, Perugia, Italy

\begin{abstract}
:
Background:

The dermis is a commonly used source tissue for biologic scaffolds; all cellular and nuclear materials need to be removed to limit the inflammatory immune response by the host organism. The decellularization is critical because it must preserve the structural integrity of the extracellular matrix. This work has analyzed a decellularization procedure commonly followed for the dermal tissue that is a chemical treatment with sodium hydroxide. The goal of this work is to identify the optimal treatment length on the basis of structural properties.
\end{abstract}

\section{Methods:}

Tensile tests have been performed on the native tissue and on tissues decellularized for 1-7 weeks in sodium hydroxide. The collected data have been analyzed through Tukey-Kramer test to assess if the mechanical properties (ultimate tensile stress and elastic modulus) of decellularized tissues were significantly different from the properties of the native tissue. These tests have been performed on specimens cut along two orthogonal directions (parallel and perpendicular to Langer's lines).

\section{Results:}

The decellularization treatment performed with sodium hydroxide in general weakens the tissue: both the ultimate stress and the elastic modulus get lower. The structural properties along Langer lines orientation are more strongly impacted, while the structural properties orthogonal to Langer lines can be preserved with an optimal duration of the decellularization treatment that is 5-6 weeks.

\section{Conclusion:}

The duration of the decellularization treatment is critical not only to reach a complete decellularization, but also to preserve the mechanical properties of the tissue; 5-6 week treatment performed with sodium hydroxide allows preserving the mechanical properties of the native tissue perpendicularly to Langer lines orientation, and minimizing the impact of the decellularization process on the mechanical properties along the Langer lines orientation.

Keywords: Acellularized dermis, allografts, decellularization treatment, elastic modulus, extracellular matrix, human dermis, tensile tests, ultimate stress.

\section{BACKGROUND}

So far, artificial scaffold-based constructs are unable to closely mimic the properties of the respective native tissues in terms of biocompatibility, function, structure, and so on. On the other hand, human-derived biomaterials preserve the

* Address correspondence to this author at the Department of Engineering, University of Perugia, Via Duranti 67, 06125 Perugia, Italy; Tel:+39 075 5853748; Fax: +39 075 5853606; E-mail: elisabetta.zanetti@unipg.it 
natural architecture from the native tissue; however, allogenic biomaterials would solicit an adverse immune response $[1,2]$. The solution is making use of decellularized allogenic material, where cells have been removed to produce a tissue as free as possible of potentially immunogenic antigens. The optimal decellularization treatment should minimize cellular and nuclear materials in the matrix, without affecting the integrity of the tissue structure, and consequently its mechanical properties [2,3]. Generally speaking, the duration of the decellularization treatment has to counterbalance two benefits: on the one hand, a longer duration gives more chance to eliminate all cellular and nuclear components; on the other hand, it could adversely influence collagen, elastin, and extracellular components.

This work focuses on acellular dermal allografts; these grafts were initially used as wound cover in burns and other large wounds [4]; however, they are now also finding numerous other clinical applications as a scaffold allowing for new tissue ingrowth: it is the case of wound healing (diabetic ulcers or burns), tendon and ligament repair [5], softtissue supplementation [6], and joint resurfacing [7]. Methods of decellularization can have different processing times and they can employ different means, eventually producing alterations of the matrix structure; the most common processes include a combination of physical, chemical, and enzymatic agents. The use of $\mathrm{NaOH}$ medium for the decellularization of human skin has been reported in the literature as an effective low-cost method, which is able to remove donor cells and hairs [8]. The dermal tissue requires a more extensive biochemical exposure and longer rinse times, like other laminated thicker tissues (e.g. cardiac muscle tissue) [3]. However, bases run the risk of decreasing the extracellular matrix (ECM) mechanical properties due to the cleavage of collagen fibrils and disruption of collagen crosslinks [1]. The authors herein have tried to elucidate to what extent this process of decellularization affects the mechanical behavior of the dermal tissue, in relation to its duration. Apposite tensile tests have been planned both along Langer lines orientation and perpendicularly, and decellularized tissue properties have been compared with the native tissue ones. As a result, indications about the optimal decellularization treatment length have been given. In literature, the tensile properties of ex vivo native skin samples have been studied by Nì Annaidh et al. [8] who calculated engineering stresses and strains, and the elastic modulus for different back areas and different orientations. They demonstrated the variability of tissue properties in relation to specimen orientation (referred to Langer lines), body area, and among different donors; therefore, the influence of the decellularization treatment length has been tested on groups of specimens coming from the same donor, with the same orientation, and belonging to the same body area. Yoder and Elliott performed tensile tests on various graft materials used in soft-tissue applications, having outlined the relevance of mechanical properties of these tissues for a successful surgical reconstruction [9]. All these authors reported ultimate engineering stresses and this might be a limit since the specimen section undergoes a significant striction during tensile tests up to rupture; therefore, true stresses have also been calculated in this work, measuring specimen section in real time.

\section{MATERIALS AND METHODS}

\subsection{Specimens}

Human reticular dermal grafts have been harvested from human donors back: the strips have been dissected, and they have been decellularized through incubation in $\mathrm{NaOH} 0.06 \mathrm{~N}$ culture medium for 1-7 weeks, at room temperature in continuous tilting, changing the medium once a week. Grafts have been finally neutralized by means of incubation in $\mathrm{HCl} 0.1 \mathrm{~N}$, obtaining human decellularized dermal matrices (HADM). HADM have been preserved at 85\% glycerol in a $4^{\circ} \mathrm{C}$ refrigerator [8], at the Turin Skin Bank. They have been used for these experiments after the approval of the Regional Transplant Centre Ethical Committee (protocol number 0006730; January 23, 2012), having obtained the written informed consent from all the study participants. Before use, HADM have been dipped for at least 3 min, and thrice in saline solution $(0.9 \%)$ at $37^{\circ} \mathrm{C}$, to eliminate all the glycerol from skin samples. The specimens have been cut parallel $\left(0^{\circ}\right)$ and orthogonal $\left(90^{\circ}\right)$ to Langer lines by means of a custom-made die cutter. The cutter has been appositely designed to prevent notches and defects, which could bias mechanical tests. On the whole, there were 3-4 specimens for each decellularization treatment length; the final specimen sizes, measured by photogrammetry, were equal to $4.33 \pm 0.57 \mathrm{~mm}$ (average \pm std width), $2.21 \pm 0.32 \mathrm{~mm}$ (average \pm std thickness), and $10.10 \pm 0.38 \mathrm{~mm}$ (average \pm std length).

Up to the instant preceding the tensile test, all samples have been kept in saline solution; during tests, they have not been hydrated since tensile tests lasted only for a few seconds.

\subsection{Photogrammetry Setup}

The size of the specimens prior to testing has been assessed through photogrammetry. A full-frame digital camera 
(Canon EOS 5D Mark II), with an autofocus lens for macro photography (Canon EF $100 \mathrm{~mm} \mathrm{f/2.8} \mathrm{Macro} \mathrm{USM)} \mathrm{has}$ been used for this aim, together with a camera stand, and two light stands. Two pictures have been taken for each specimen: the top view and the side view; the respective 21.0 MP digitized images have been analyzed by means of ImageJ software. The resolution was equal to $0.01 \mathrm{~mm} /$ pixel.

During mechanical tests, one more camera with its tripod (Canon EOS 400D) has been added to obtain both specimen width and thickness in real time; thanks to apposite capture software (DSLR Remote Pro).

\subsection{Mechanical Tests}

Specimens have been clamped to the Bose Electroforce ${ }^{\circledR}$ testing machine; some preliminary tests have been performed to optimize the gripping force, avoiding specimen slippage without damaging the tissue integrity. Rupture tensile tests have been performed for all samples in displacement control at a strain rate equal to $0.032 \mathrm{~s}^{-1}$. The initial gap between machine clamps was equal to $5 \mathrm{~mm}$. None of the specimens was ruptured near the clamps.

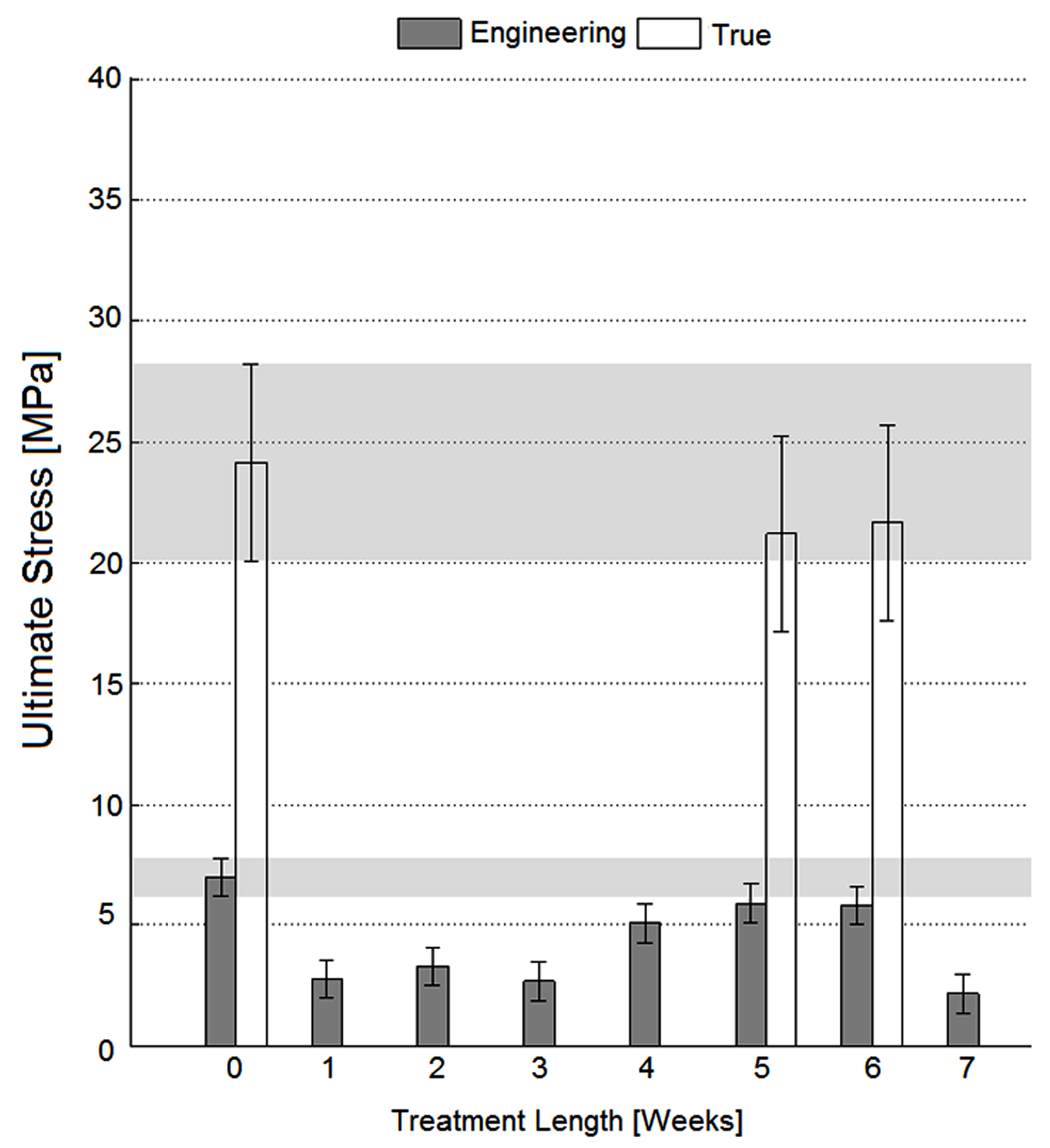

Fig. (1). Ultimate stress for specimens perpendicular to Langer lines; error bars refer to Tukey-Kramer test intervals. According to this test, overlapping intervals are not significantly different; therefore, specimens decellularized for 5-6 weeks have an ultimate stress close to the native dermis ones considering both engineering and true stresses $(p<0.05)$. 


\subsection{Elaboration Data}

The ultimate tensile stresses have been reported in different forms to allow making comparisons with other data in the literature. Engineering stresses have been calculated by dividing the load with the initial specimen area $\mathrm{A}_{0}$ [9], while true stresses have been calculated by dividing the load with the current specimen area $A_{i}[10]$. Specimen images (frontal and lateral views) have been sampled every $3 \mathrm{~s}$ by the aforementioned digital cameras to calculate the actual specimen section. The true specimen area has been calculated for untreated specimens and for specimens treated for 5-6 weeks since the results obtained on these specimens justified performing more detailed inquiries, as detailed in the following.

Young's modulus (E) has been calculated from the linear portion of the stress-strain curve [11]: curve data have been locally derived with a moving average linear regression, and the constant trend of the derived curve has been considered.

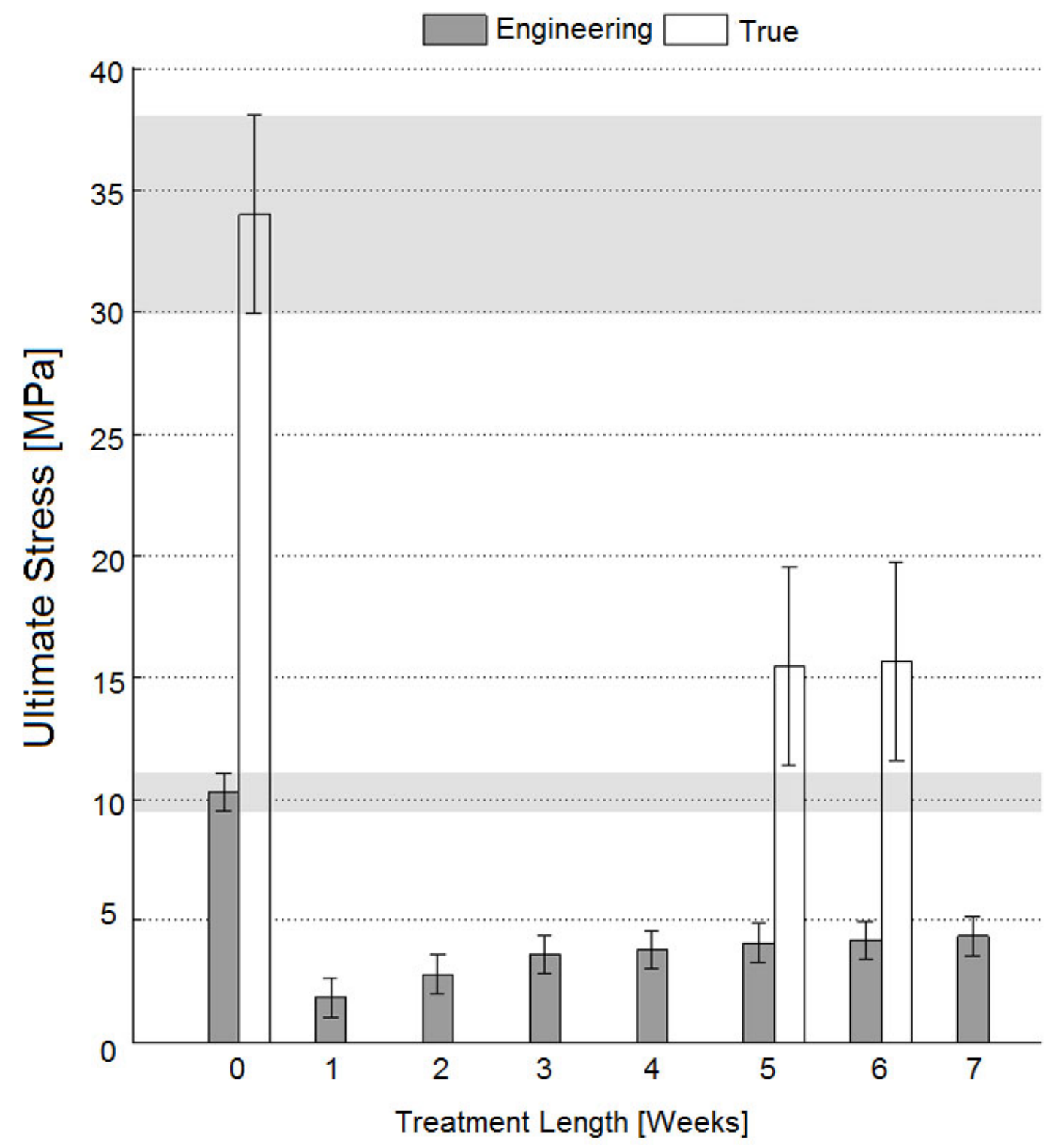

Fig. (2). Ultimate stress for specimens parallel to Langer lines; error bars refer to Tukey-Kramer test intervals. According to this test, none of the specimens has an ultimate stress as high as the native dermis ones $(p<0.05)$.

\subsection{Statistical Analysis of Data}

The mechanical properties of the dermis have been reported in relation to the duration of the decellularization treatment (from 0 to 7 weeks with a step size of 1 week, called T0, T1, .., T7 in the following) for both sample 
orientations $\left(0^{\circ}\right.$ and $90^{\circ}$ with reference to Langer lines).

The normality of the statistical distribution of all parameters has been preventively checked by lillie-test function. Tukey-Kramer post hoc test has been used to compare properties obtained for different durations of the decellularization treatment versus the initial tissue properties (T0- $0^{\circ}$ and $\mathrm{T} 0-90^{\circ}$, respectively). The significance level has been set equal to 0.05 for all tests.

\section{RESULTS AND DISCUSSION}

Results concerning the ultimate tensile stress have been reported in Fig. (1) and Fig. (2), respectively, for specimens cut parallel and perpendicular to Langer lines, while results concerning the elastic modulus have been reported in Fig. (3) and Fig. (4), respectively, for $0^{\circ}$ and $90^{\circ}$ specimens. The comparison of mechanical properties measured on specimens parallel or perpendicular to the Langer lines is in accordance with the well-known dermal tissue anisotropy, and is related to the prevalent orientation of collagen fibers [9, 11]: with reference to data preceding decellularization treatment ("T0"), specimens cut along Langer lines have exhibited a higher ultimate stress (33.95 MPa true stresses, and 10.28 $\mathrm{MPa}$ engineering stress) and a higher elastic modulus $(21.8 \mathrm{MPa})$ when compared with specimens cut perpendicularly to Langer lines (24.11 MPa ultimate true stresses, 7.01 MPa ultimate engineering stress, and $12.31 \mathrm{MPa}$ elastic modulus)

(Figs. 1 and 2) testify that referring to engineering stress would lead to a considerable underestimation of the ultimate stress for tests like those performed here (white columns are always higher than grey columns), due to the significant specimen striction taking place before specimen rupture, and to the small section of the specimens tested here (the thickness of dermal tissue is lower than $3 \mathrm{~mm}$ ). Nevertheless, the patterns of ultimate stress versus decellularization treatment length have been found to be consistent between engineering and true stress formulations for the tensile tests (5-6 weeks values are similar and are smaller than 'T0' values both in Figs. 1 and 2).

All pictures testify that the decellularization treatment set up with sodium hydroxide has weakened the mechanical properties of the tissue ('T0' values remain the highest for whatever treatment length both in Figs. (1) and (2)), and its effect is more deleterious for specimens cut along Langer line orientation where collagen fiber bundles are stressed more closely to their axes (the difference between 'T0' values and all treated specimens is higher in Fig. (2) compared with Fig. (1)). Effectively, it is known that alkaline bases are able to degrade the structural components of the matrix including collagen to some extent: Sheridan et al. [12] used sonication and $0.5 \mathrm{M} \mathrm{NaOH}$ to controllably degrade small collagen fibrils of decellularized pig arterial tissue; in more detail, bases reduce the mechanical properties due to the cleavage of collagen fibrils and disruption of collagen nonreducible crosslinks [13].

However, all results have demonstrated a partial recovery of mechanical properties for longer treatment lengths (with specific reference to the decellularization treatment performed here through incubation in $\mathrm{NaOH} 0.06 \mathrm{~N}$ culture medium at room temperature, in continuous tilting): according to Fig. (1), the ultimate engineering tensile stress of untreated tissues cut perpendicular to Langer lines is not significantly different from those of specimens treated for 5 weeks (Tukey-Kramer test, $p<0.05$ ), or for 6 weeks (Tukey-Kramer test, $p<0.05$ ). These observations have induced one to perform more detailed analyses where real-time specimen width and thickness have been acquired to calculate true stresses. These tests have further confirmed the similarity between untreated specimens and specimens treated for 5-6 weeks through incubation in $\mathrm{NaOH} 0.06 \mathrm{~N}$ in continuous tilting (Fig. 1, 'T0' and 'T5-6' white columns). On the contrary, the ultimate tensile stress of tissues cut along Langer line orientation has got significantly lower, for whatever treatment duration (Fig. 2, 'T0' and 'T5-6' grey columns), and this observation has also been confirmed referring to true stresses (Fig. 2, 'T0' and 'T5-6' white columns). The observation of results concerning the elastic modulus (and consequently to physiologic stress levels) has led to similar conclusions: with reference to $90^{\circ}$ orientation (Fig. 3), the performance of specimens treated for 5-6 weeks in $\mathrm{NaOH} 0.06 \mathrm{~N}$ culture medium is again similar to the performance of untreated specimens ('T5-6' vs. 'T0', Fig. 3); however, specimens treated for 4 weeks ('T4' in Fig. 3) are most similar to untreated specimens. With reference to Langer line orientation (Fig. 4), whatever treatment length has degraded the tissue mechanical properties ('T0' column is the highest in Fig. 4), 5 and 7 week treatments in $\mathrm{NaOH} 0.06 \mathrm{~N}$ culture medium are slightly more conservative ('T5' and 'T7' in Fig. 4). In summary, the experiments have demonstrated that there is an improvement in the mechanical properties mainly along $90^{\circ}$ orientation for decellularization treatments in sodium hydroxide lasting 5-6 weeks (Fig. 3): this result can be explained assuming the recovery of intermolecular interactions between low-order collagen structures, resulting in fiber reassembly (the reversible dissociation of collagen has been clearly demonstrated [14]). 


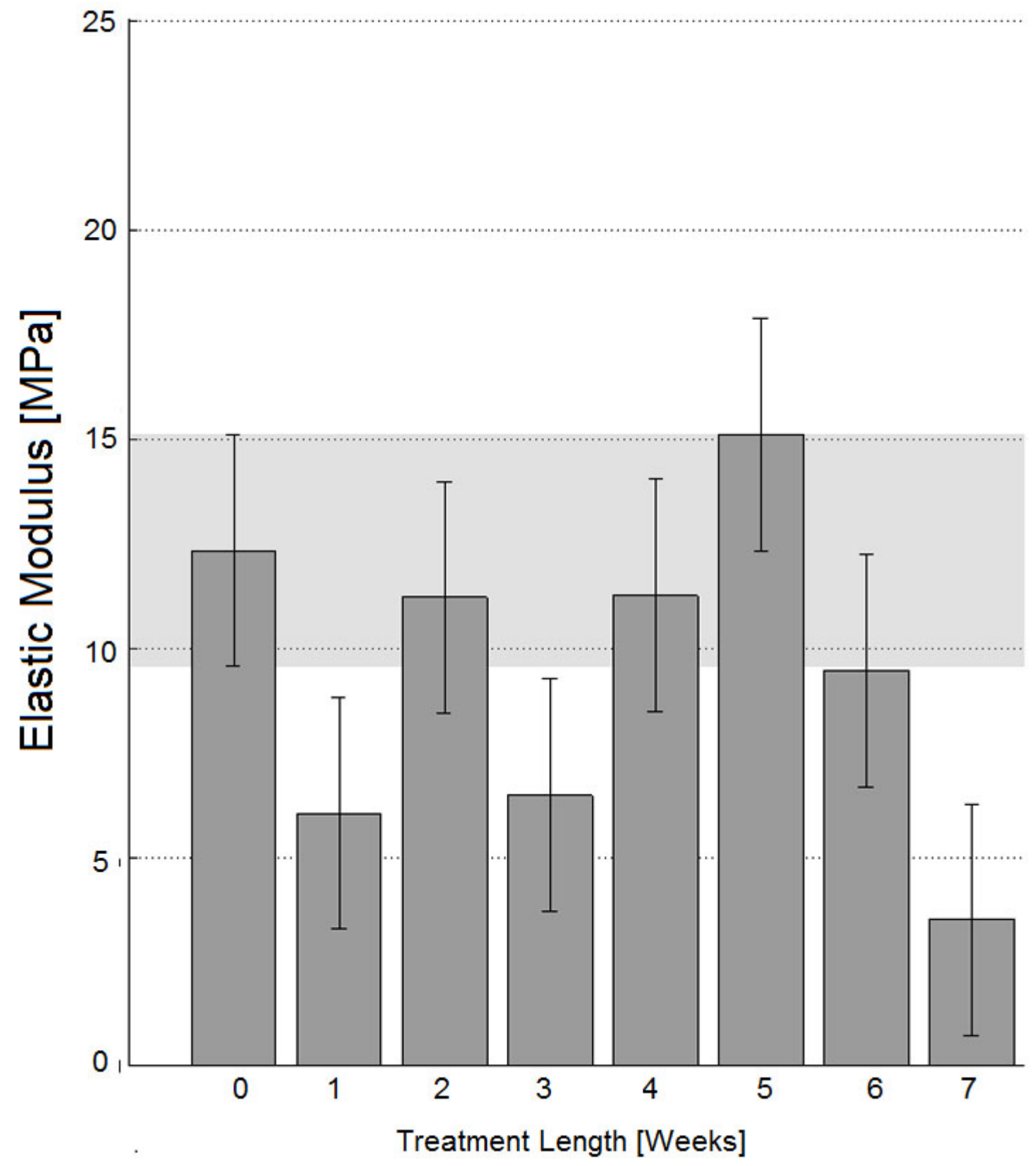

Fig. (3). Elastic modulus for specimens perpendicular to Langer lines; error bars refer to Tukey-Kramer test intervals. According to this test, specimens decellularized for 2, 4-6 weeks have an elastic modulus close to the native dermis one $(p<0.05)$.

Other authors agree with the final findings of the present experimental work: they evaluated a cost-effective method based on low concentrations of $\mathrm{NaOH}$ for the decellularization of human donor skin preserved in $85 \%$ glycerol [10] and found that a 6 -week incubation period was optimal. It should be stressed how decellularization through chemical agents is commonly considered to be a cost-effective method [10, 15]; nevertheless, its long duration could have inherent problems from a commercialization standpoint.

Differences of behaviors between $0^{\circ}$ and $90^{\circ}$ orientation samples (Fig. 1 vs. Fig. 2, and Fig. 3 vs. Fig. 4) can be explained considering that $0^{\circ}$ samples are stressed along the main collagen fibers orientation, and are therefore more sensitive to collagen molecules denaturation, cleavage, or partial disruption, which can take place during treatments in alkaline culture media.

The obtained results refer to "ex vivo" tests on pre-implant specimens; when these grafts are implanted, remodeling processes take place, and tensile mechanical properties are likely to undergo significant variations; some clues can be gained from the functional tests performed in a rat subcutaneous implant model and in a porcine transplantation model 
by Richters et al. [10]. In future, it would be very interesting to perform in vivo tensile tests [16, 17] on implanted dermal substitutes.

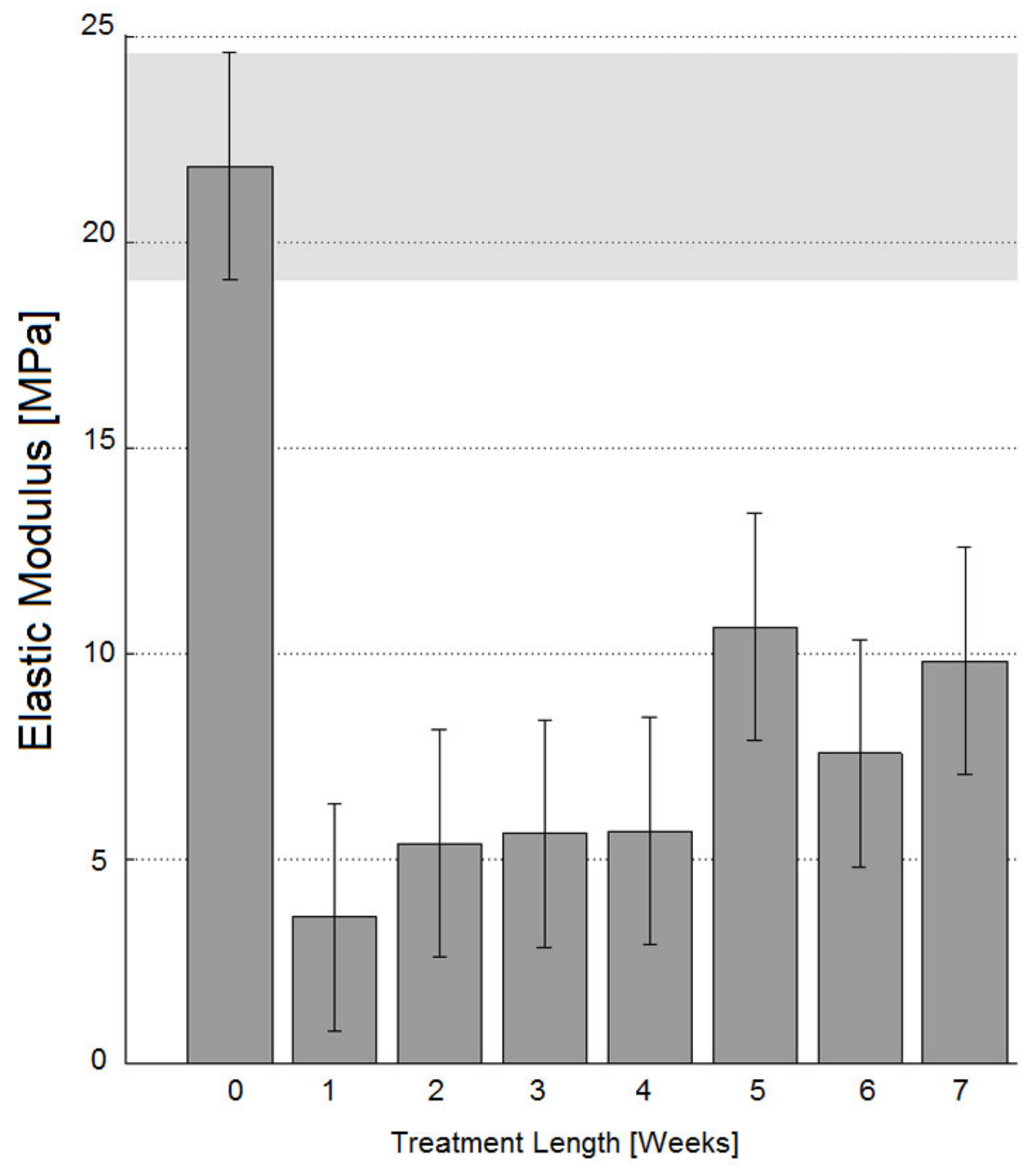

Fig. (4). Elastic modulus for specimens parallel to Langer lines; error bars refer to Tukey-Kramer test intervals. According to this test, none of the specimens has an elastic modulus as high as the native dermis one $(p<0.05)$.

\section{CONCLUSION}

This work has focused on the study of the consequences of an alkaline decellularization process (incubation in $\mathrm{NaOH} 0.06 \mathrm{~N}$ culture medium for 1-7 weeks, at room temperature in continuous tilting) on the structural function of dermal tissue, referring to the tissue strength (that is the ultimate stress measured during tensile tests at 3.2\%/s strain rate), and to the tissue behavior at physiologic stress levels (elastic modulus of the linear portion of stress/strain curve).

The mechanical properties of the decellularized tissue have been compared with those of the native tissue, and considering two orthogonal orientations (parallel or perpendicular to the main orientation of collagen fibers), for different lengths of the decellularization treatment.

According to the results, the optimal duration of the decellularization treatment in $\mathrm{NaOH}$ is $5-6$ weeks, since these treatment lengths have produced the most similar results to the native tissue. 
The reliable quantitative measurement of structural properties of dermal tissue up to rupture requires an experimental setup able to capture specimen geometry variations in real time, while engineering stress and strain data have resulted in providing significant indication for comparative analyses among different decellularization treatment lengths.

\section{CONFLICT OF INTEREST}

The authors confirm that this article content has no conflict of interest.

\section{ACKNOWLEDGEMENTS}

Declared none.

\section{REFERENCES}

[1] P.M. Crapo, T.W. Gilbert, and S.F. Badylak, "An overview of tissue and whole organ decellularization processes", Biomaterials, vol. 32, no. 12, pp. 3233-3243, 2011. [http://dx.doi.org/10.1016/j.biomaterials.2011.01.057] [PMID: 21296410]

[2] T.J. Keane, I.T. Swinehart, and S.F. Badylak, "Methods of tissue decellularization used for preparation of biologic scaffolds and in vivo relevance", Methods, vol. 84, pp. 25-34, 2015.

[http://dx.doi.org/10.1016/j.ymeth.2015.03.005] [PMID: 25791470]

[3] T.W. Gilbert, T.L. Sellaro, and S.F. Badylak, "Decellularization of tissues and organs", Biomaterials, vol. 27, no. 19, pp. 3675-3683, 2006. [PMID: 16519932]

[4] M.L. Iorio, J. Shuck, and C.E. Attinger, "Wound healing in the upper and lower extremities: a systematic review on the use of acellular dermal matrices", Plast. Reconstr. Surg., vol. 130, no. 5, suppl. 2, pp. 232S-241S, 2012. [http://dx.doi.org/10.1097/PRS.0b013e3182615703] [PMID: 23096978]

[5] D.C. Acevedo, B. Shore, and R. Mirzayan, "Orthopedic applications of acellular human dermal allograft for shoulder and elbow surgery", Orthop. Clin. North Am., vol. 46, no. 3, pp. 377-388, x, 2015. [http://dx.doi.org/10.1016/j.ocl.2015.02.006] [PMID: 26043051]

[6] S.L. de Souza, A.B. Novaes Jr, D.C. Grisi, M. Taba Jr, M.F. Grisi, and P.F. de Andrade, "Comparative clinical study of a subepithelial connective tissue graft and acellular dermal matrix graft for the treatment of gingival recessions: six- to 12-month changes", J. Int. Acad. Periodontol., vol. 10, no. 3, pp. 87-94, 2008. [PMID: 18714934]

[7] U.G. Longo, A. Berton, S. Alexander, N. Maffulli, A.L. Wallace, and V. Denaro, "Biological resurfacing for early osteoarthritis of the shoulder", Sports Med. Arthrosc. Rev., vol. 19, no. 4, pp. 380-394, 2011. [http://dx.doi.org/10.1097/JSA.0b013e318211c473] [PMID: 22089288]

[8] A. Ní Annaidh, K. Bruyère, M. Destrade, M.D. Gilchrist, and M. Otténio, "Characterization of the anisotropic mechanical properties of excised human skin", J. Mech. Behav. Biomed. Mater., vol. 5, no. 1, pp. 139-148, 2012. [http://dx.doi.org/10.1016/j.jmbbm.2011.08.016] [PMID: 22100088]

[9] J.H. Yoder, and D.M. Elliott, "Nonlinear and anisotropic tensile properties of graft materials used in soft tissue applications", Clin. Biomech. (Bristol, Avon), vol. 25, no. 4, pp. 378-382, 2010. [http://dx.doi.org/10.1016/j.clinbiomech.2010.01.004] [PMID: 20129728]

[10] C.D. Richters, A. Pirayesh, H. Hoeksema, E.W. Kamperdijk, R.W. Kreis, R.P. Dutrieux, S. Monstrey, and M.J. Hoekstra, "Development of a dermal matrix from glycerol preserved allogeneic skin", Cell Tissue Bank., vol. 9, no. 4, pp. 309-315, 2008. [http://dx.doi.org/10.1007/s10561-008-9073-4] [PMID: 18491218]

[11] A. Ní Annaidh, K. Bruyère, M. Destrade, M.D. Gilchrist, C. Maurini, M. Otténio, and G. Saccomandi, "Automated estimation of collagen fibre dispersion in the dermis and its contribution to the anisotropic behaviour of skin", Ann. Biomed. Eng., vol. 40, no. 8, pp. 1666-1678, 2012 .

[http://dx.doi.org/10.1007/s10439-012-0542-3] [PMID: 22427196]

[12] W.S. Sheridan, G.P. Duffy, and B.P. Murphy, "Mechanical characterization of a customized decellularized scaffold for vascular tissue engineering", J. Mech. Behav. Biomed. Mater., vol. 8, pp. 58-70, 2012. [http://dx.doi.org/10.1016/j.jmbbm.2011.12.003] [PMID: 22402154]

[13] J.S. Cartmell, and M.G. Dunn, "Effect of chemical treatments on tendon cellularity and mechanical properties", J. Biomed. Mater. Res., vol. 49, no. 1, pp. 134-140, 2000. [http://dx.doi.org/10.1002/(SICI)1097-4636(200001)49:1<134::AID-JBM17>3.0.CO;2-D] [PMID: 10559756]

[14] A.T. Yeh, B. Choi, J.S. Nelson, and B.J. Tromberg, "Reversible dissociation of collagen in tissues", J. Invest. Dermatol., vol. 121, no. 6, pp. 1332-1335, 2003.

[http://dx.doi.org/10.1046/j.1523-1747.2003.12634.x] [PMID: 14675178]

[15] T.H. Petersen, E.A. Calle, M.B. Colehour, and L.E. Niklason, "Matrix composition and mechanics of decellularized lung scaffolds", Cells Tissues Organs (Print), vol. 195, no. 3, pp. 222-231, 2012. 
[http://dx.doi.org/10.1159/000324896] [PMID: 21502745]

[16] C. Flynn, A. Taberner, and P. Nielsen, "Modeling the mechanical response of in vivo human skin under a rich set of deformations", Ann. Biomed. Eng., vol. 39, no. 7, pp. 1935-1946, 2011. [http://dx.doi.org/10.1007/s10439-011-0292-7] [PMID: 21394556]

[17] F. Khatyr, C. Imberdis, P. Vescovo, D. Varchon, and J-M. Lagarde, "Model of the viscoelastic behaviour of skin in vivo and study of anisotropy", Skin Res. Technol., vol. 10, no. 2, pp. 96-103, 2004.

[http://dx.doi.org/10.1111/j.1600-0846.2004.00057.x] [PMID: 15059176]

Received: October 12, 2015

Revised: December 17, 2015

Accepted: February 17, 2016

(C) Terzini et al.; Licensee Bentham Open.

This is an open access article licensed under the terms of the Creative Commons Attribution-Non-Commercial 4.0 International Public License (CC BY-NC 4.0) (https://creativecommons.org/licenses/by-nc/4.0/legalcode), which permits unrestricted, non-commercial use, distribution and reproduction in any medium, provided the work is properly cited. 